\title{
Author Correction: Scaling out Ising machines using a multi-chip architecture for simulated bifurcation
}

\author{
Kosuke Tatsumura (D), Masaya Yamasaki (D) and Hayato Goto (D)
}

Correction to: Nature Electronics https://doi.org/10.1038/s41928-021-00546-4, published online 1 March 2021.

In the Supplementary Information file originally published with this Article, it was unclear where the nth pseudorandom number should be used in the $N \times N$ weight matrix of the MAX-CUT problem. Thus, the sentence " $\mathrm{K}_{N}(N=\{4,096,8,192,16,384\})$ is an $N$-node complete-graph MAX-CUT problem whose weights are defined similarly as for $\mathrm{K}_{2000}: w_{i j}=w_{j i}=1-2 r_{f(i, j)}(i>j)$, where $f(i, j)=(i-1)$ $(i-2) / 2+j$ and $r_{n}$ is the lowest bit, $\{0,1\}$, of the $n$th random number generated by a pseudorandom number algorithm called the Mersenne Twister [4] with seed $=1$." has been corrected to " $\mathrm{K}_{N}(N=\{4,096,8,192,16,384\})$ is an $N$-node complete-graph MAX-CUT problem whose weights are defined similarly as for $\mathrm{K}_{2000}: w_{i j}=w_{j i}=2 r_{f(i, j)}-1(i<j)$, where $f(i, j)=N(i-1)-i(i-1) / 2+(j-i)$ and $r_{n}$ is the lowest bit, $\{0,1\}$, of the $n$th random integer generated by the pseudorandom integer generation function in the algorithm called the Mersenne Twister [4] with seed = 1." The amended Supplementary Information file has now been uploaded to the Article.

Published online: 27 July 2021

https://doi.org/10.1038/s41928-021-00637-2

(C) The Author(s), under exclusive licence to Springer Nature Limited 2021 